\title{
The Application of Autonomous Learning to Fostering Cross-cultural Communication Competence* $^{*}$
}

\author{
Fangpeng Gai \\ School of Foreign Language Studies, Shandong Jiaotong University, China
}

\begin{abstract}
The significance of autonomous learning has been proved theoretically and practically both at home and abroad in recent years. We have also been putting great emphasis on the learner's autonomy in our college English teaching in order to encourage the students to actively participate in the learning process, but due to various reasons, the cultivation of cross-cultural competence, which has become a critical teaching objective has not been paid due attention during not only classroom lecturing but also after-class activities. Based on the questionnaire survey among the author's students on the status quo of cross-cultural teaching and learning, this paper proposes ways of autonomous learning that the students may adopt to improve their cross-cultural communication competence and the teacher's role as well.
\end{abstract}

\section{Index Terms - autonomous learning, cross-cultural communication, College English teaching}

Communication is the ultimate aim of language teaching, so we should attach unprecedentedly greater importance to the role of the learner or the communicator, which demands us to re-locate the roles of the teacher and student during the process of foreign language teaching in order to fully mobilize the student's enthusiasm and initiatives to get totally involved to shift our classroom teaching from a teacher-centered mode to a student-centered one to achieve the teaching goal mainly depending on leaner's autonomy. From the long run, what is more significant is that the cultivation of autonomous learning can lay a sound and solid foundation for their post-school education and lifelong learning.

\section{Autonomous LeARning}

Nowadays we have different ways of learning such as "self-education", "self-access", "self-instruction", "distance learning" and etc, all of which indicate the way that one takes to learn, yet different from the above the term autonomous learning, which is now a buzzword in the foreign language teaching field, mainly focuses on the ability or capacity that somebody has to control over or take charge of individual's learning.

Holec introduced the concept of "autonomous learning" in Autonomy and Foreign Language Learning in 1981, and he defined it from five aspects: (1) determining objectives, (2) defining content and progressions, (3) selecting methods and techniques to be used, (4) monitoring procedure of acquisition and (5) evaluating what has happened. According to Benson \& Voller (1997), it should cover five elements when talking about autonomous learning: (1) for situations in which learners study entirely on their own, (2) for a set of skills which can be learned and applied in self-directed learning, (3) for an inborn capacity which is suppressed by institutional education, (4) for the exercise of learner's responsibility for their own learning and (5) for the right of learners to determine the direction of their own learning.

After that, a great deal has been done to define what autonomous learning is. Dickinson (1987) argues "situation in which the learner is totally responsible for all of the decisions concerned with his/her learning and the implementation of these decisions". Some domestic scholars also conduct their research and give their understanding of this term. For example, Cheng Xiaotang defines it in the following way: (1) it is an intrinsic mechanism comprehensively formed by learner's attitude, aptitude and learning strategy, (2) it is the free choice of learner's objective, learning content, and learning methodology and material, and (3) it is a learning mode tailored to learner's need, in the mean time, directed and affected by the entire teaching objectives and teacher's guidance.

Although an accurate and widely accepted definition of autonomous learning has not been concluded by far, the common core components can be drawn from above: (1) generate one's own learning goal, (2) set one's own learning plan, (3) choose one's own learning methodology, (4) monitor one's own learning process, (5) self-evaluate one's own learning progression and (6) adjust one's learning strategy according to the evaluation. Autonomous learning is the way how one learns with a special concern of whether the learning is driven, controlled, assessed and managed by oneself. To be specific, if a student's learning motivation is self-driven, learning contents, materials and strategies are self-chosen, learning process is self-regulated, learning outcome is self-evaluated, we can say his/her learning is autonomous. Otherwise, if a students' learning motivation, learning contents, materials, strategies and etc. are mainly

\footnotetext{
* Supported by Research Program of China Institute of Communications Education (1202-174) and Social Science Research Program of Shandong Province (12CWJZ03).
} 
depending on other's guidance and regulation, then his/her learning is passive and non-autonomous.

\section{Cross-cultural Communication}

Language is the carrier of and verbal expression of one certain culture and culture is the specific set of beliefs, ideas, customs and norms of a community with a distinct language, which means all the ways in which a group of people act, dress, think, feel and etc.

Cross-cultural communication, also frequently referred to as intercultural communication describes the ability to successfully form, foster, and improve relationships with members of a culture different from one's own. It is based on knowledge of many factors, such as the other culture's values, perceptions, manners, social structure, and decision-making practices, and an understanding of how members of the group communicate--verbally, non-verbally, in person, in writing, and in various business and social contexts, to name but a few. At the same time, cross-cultural communication is a field of study that looks at how people from differing cultural backgrounds communicate, in similar and different ways among themselves, and how they endeavor to communicate across cultures. In fact, simply speaking, it is the communication happened between a native speaker and a non-native speaker of a certain language, and from a broader sense, it can be the communication between speakers who have cultural differences no matter whether they are fellow countrymen or not. So we should always bear in mind of the cultural differences when communicating with non-native speakers for in the context of cross-cultural communication misunderstanding often happens resulted from ambiguous understanding of the target culture, and sometimes it may cause failure in communication because people from different cultural backgrounds are always encoding and decoding information differently based on their previously acquired communication patterns, increasing the chances of misunderstanding and failure.

But the reality is that with the globalization cross-cultural communication, which is becoming more and more popular and an inevitable exercise still remains a big challenge for many people. In order to meet the needs of the society, the cultivation of cross-cultural communication has been listed as one of the teaching objectives in College English Curriculum Requirements 2007, which stated "College English teaching should be a comprehensive teaching system of teaching mode and methodology, which is guided by foreign language teaching theories and takes English language knowledge, application techniques, cross-cultural communication competence and learning strategies as its main contents."

But what is cross-cultural communication competence? It is the ability or capacity of communicating with others who have cultural differences from you. According to Spitzberg, there are three issues that can be taken as the components of cross-culture communication competence: motivation, communication knowledge and skills. Motivation is defined as the process that initiates and guides goal-oriented behaviors which involves the biological, emotional, social and cognitive forces that activate behavior, or it can be simply used to describe why a person does something. So here, the motivation is the willingness to conduct cross-cultural communication. Spitzberg and Cupach (1984) propose that communication competence has two criteria: appropriateness and effectiveness. "Appropriateness" refers to whether the behaviors can be accepted or considered as proper in the target culture, and "effectiveness" refers to whether the communicators achieve their desired communicating result or to what degree they do that.

\section{Status Quo of Cross-cultural Communication Learning}

In order to get overall and objective understandings of the cross-cultural communication learning situation of non-English majors in our university, the author carries out a questionnaire survey among randomly selected 76 students of majors of civil engineering, applied physics, computing science and 70 of them return their questionnaire and feedback. The survey covers three parts: personal information, status quo of cross-cultural communication learning and application of cross-cultural communication knowledge.

The result shows that all the students think language and culture has a very close relationship and culture teaching will do good to their English language learning. 45.7\% of the respondents assume that they do not have much knowledge of cross-cultural communication and much of their acquisition is something about geography and historical knowledge of those major English-speaking countries such as the USA, the UK, Canada and etc. 52.9\% of them admit that their English teacher sometimes carries out culture teaching but much is done at the leading-in stage. $57.1 \%$ of them say English movies and the Internet are the two major resources of their cross-culture communication knowledge.

When it comes to the willingness of cross-cultural communication, only $21.4 \%$ of the respondents say that they feel relaxed and easy when communicating with foreigners while $65.7 \%$ of them say that they are nervous or very nervous and hardly can express themselves clearly and accurately. The main obstacles resulting in their unsuccessful communication mainly include their difficulty in understanding of the idioms, their small vocabulary and their nonstandard pronunciation.

In the application of cross-cultural communication knowledge, most of the students can understand the general etiquette, but it is hard for them to explain the reason behind the misunderstanding occurring in cross-cultural communication. For example, a Chinese girl Xiaorui and an American girl Anny are good friends who help each other a lot both in and after school. But Anny never invites Xiaorui to come to her home in spite of their passing by her home many times. Xiaorui asks Anny for help when the family makes a move, but it seems that Anny is impatient when 
Xiaomei makes a phone call to her. This confuses Xiaorui, for in her mind, being good friends they should help each other. Many respondents argue that Anny does not take Xiaorui as her friend at all. In fact, the main reason is that we have different conception about friendship. To us, being good friends means we should help each other anytime, anywhere when possible, even at any price. On the contrary, western people attach great importance to privacy demanding making an appointment in advance no matter how close friends or relatives they are. These kinds of misunderstanding caused by cultural difference are very common in cross-cultural communication.

\section{Autonomous Learning Strategies to Improve Cross-Cultural COMmunication Competence}

\section{Teacher's role}

To implement autonomous learning does not simply diminish teacher's responsibilities; Wright (1987) thinks that the teachers' roles are multiple in autonomous learning situations. Transmitting language knowledge is the primary responsibility that a teacher should fulfill, but what is more critical to the teacher during the process of autonomous learning is to play more roles as facilitators, coordinators, organizers and assessors, which means during the process the teachers are supposed to identify the clear teaching goals of the course to the students, help the students to set their own goals, choose the learning content and strategy, select learning materials and evaluate their learning outcome. These kinds of intervention from the teacher can be classified as positive guidance. And there is other type of intervention which can be interpreted as prohibitive intervention which means the intervention from the teacher under some circumstances when the teacher finds there are some serious problems which may cause great barrier to his/her autonomous learning that the student is not aware of or is not willing to correct. For example, when the teacher finds one student does not pay full attention to his/her study and often cuts class, the teacher may take some proper actions like leaving appropriate level of difficulty assignment to him/her to bring him/her back on track. But comparatively speaking, this positive guidance should be the more preferable help that the teacher should offer especially at an early stage when the students feel at a loss not knowing what exactly should be done to implement autonomous learning.

To facilitate the students' autonomous learning, some adjustments also should be made to our existing way of course evaluation. Now, we mainly take the method of summative evaluation in our college English teaching at the end of each semester, which usually happens in a limited time and relies on scores to evaluate the students' learning outcome. In order to reduce the anxiety and nervousness caused by summative evaluation and guide the students to concentrate on the learning process rather the exam itself, formative evaluation should be adopted. This is a key step guiding the students to foster their autonomous learning.

The shift from a teacher-centered mode to a student-centered mode is not only a change of name but calls on more dedication from the teacher in order to encourage the students to take more control over their learning by themselves to play the most and best of their initiatives rather than sit in the classroom to listen to the teacher and take notes passively, which is always the situation we are experiencing every day.

\section{Strategies for the students}

Wenden (1998) concludes seven roles of autonomous learners in aspect of language learning: 1). Autonomous learners have insights into their learning styles and strategies; 2). Autonomous learners take an active approach to the learning task at hand; 3). Autonomous learners are willing to take risks, i.e. to communicate in the target language at all costs; 4). Autonomous learners are good guessers; 5). Autonomous learners attend to form as well as content, that is, place importance on accuracy as well as appropriateness; 6). Autonomous learners develop the target language into a separate reference system and are willing to revise and reject hypotheses and rules that do not apply; 7). Autonomous learners have a tolerant and outgoing approach to the target language.

These are the typical and shared characteristics among the autonomous learners, and when it comes to the college students who implement this approach to improve their cross-cultural communication competence, the following suggestions may be taken into consideration.

1) Maintain strong learning motivations

In fact, whether having strong learning motivations is an important factor in determining a student's learning is autonomous or not; that is, if lacking intrinsic learning motivations, a student will not really take responsibilities for his/her learning. Only by generating strong motivations can a student consciously identify his/her learning needs, select his/her learning strategies and finally learn to learn. Pintrich argues that there are three types of motivational beliefs: (1) self-efficacy beliefs (that is, judgment of one's capabilities to do the academic tasks), (2) task value beliefs (that is, beliefs about the importance of, interest in, and value of the task), and (3) goal orientations (that is, whether the focus is on mastery and learning of the task, grades or extrinsic reasons for doing the task, or relative ability in relation to social comparisons with other students).

Self-efficacy is one's confidence of capability of doing something. In practice, a student should have confidence in accomplishing the teaching goals of our cross-cultural communication course through his/her own hard work, the teacher's guidance and peer support. Students of high self-efficacy are more likely to have better academic performance than those of comparatively low self-efficacy for the better planning, monitoring, assessing and adjusting.

Eccles (1983) has proposed the three components of task value: the individual's perception of the importance of the task, their personal interest in the task, and their perception of the utility value of the task for future goals. When it comes to the cross-cultural communication competence, the student should have a full and adequate understanding of 
the importance of cross-cultural competence as a qualified citizen of the times of globalization. From a utilitarian perspective, the cross-cultural competence can be useful for them immediately (e.g., it can help them to freely communicate with the foreign teachers and students) or in the future (e.g., being competent in cross-cultural communication may help them to find a better job).

2) Set individual goals according to one's own situation

At this stage, the first thing for the teacher to do is to clearly explain the teaching goals of the course to the students, for the explicit teaching goals can set a sound foundation for the students to select their own goals and content. As is known, most of the students are driven to learn by their parents and the teachers before they enter college, and the result of this long term situation is that many of the students cannot effectively manage their learning depending on themselves, which attaches great importance to the teacher's guidance to change their learning concept, take charge of their learning and eventually achieve autonomous learning. So, based on the teaching goals of the course, the teacher should help the students to identify their individual learning expectations through discussion, questionnaire, quiz and other possible ways.

Then the students should set their individual learning goals after each one has an objective understanding of his/her own level. But here the learning goals can be divided into two categories: long-term goals and short-term goals. Long-term goals refer to those that can only be achieved or accomplished after a comparatively long time of effort such as semester goals, curriculum goals and academic goals, while short-term goals are those that are set up for some specific time or unit. When setting the long-term goals or the short-term goals, the students should be reminded of the level of goal difficulty, that is, the goals should be moderate or somewhat challenging which means in order to achieve the goals he or she has to take some effort. If the student can accomplish the goals without too much effort, the goals fall into being so easy that the driving force of autonomous learning will not last for a longer time, and thus autonomous learning cannot be implemented. On the other hand, if the goals are obviously beyond a specific student's ability and too difficult to achieve, it would be impossible for the student to stick to the goals for a long time, because his/her effort turns out to be in vain no matter how hard he/she works. And this can be clearly shown in the following figure.

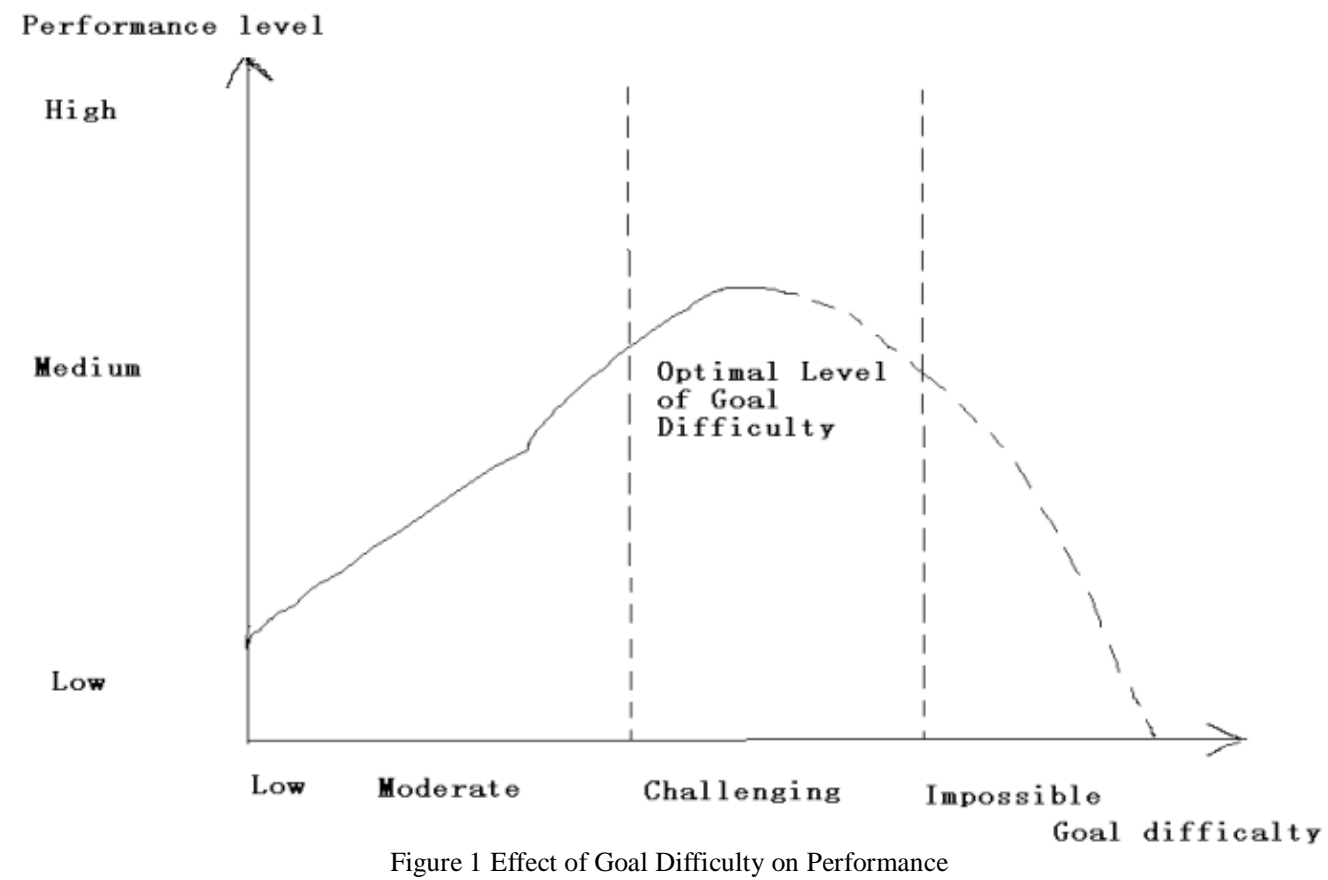

\section{3) Select preferred learning strategies}

Learning strategies refer to those ideas or actions that help us to acquire new information or knowledge based on our acquired knowledge which can be displayed in different forms such as outlining, note-taking, summarizing, paraphrasing and etc. Weinstein (1987) concludes all the strategies into 5 categories that can be used in fostering autonomous learning: rehearsal strategies, elaborating strategies, organization strategies, comprehension monitoring strategies, and affective strategies.

In terms of individual's learning methods, it should be determined by comprehensive factors such as one's learning level, learning goals, and learning content as well, which means it is unimaginable to figure out a fixed and universal learning method favorable for each one, and at the same time, it is impossible for anyone to stick to one category of strategy from beginning to the end. Different components of cross-cultural communication competence call for different learning strategies: for the part of knowledge, it should be acquired mainly through learning either by reading, writing or reciting and etc; for the part of skill, it only can be acquired through practice; for the part of motivation, it should be 
maintained and promoted through practice which means the situation when one puts his knowledge into practice and get the result he/she has imagined will help one keep high motivation.

\section{4) Assess learning by oneself}

In the long practice of assessment in our education from primary school to college, the full responsibility of assessing the students' learning and outcome mainly goes to the teacher which leaves almost no chance for the students to be involved in the assessment, and among the assessment tools, test with scores is the most widely used one which results in a side-effect of the situation of most students' and their parents' focus only on the score itself and paying insufficient attention to the teacher's feedback either orally or written. Different from our traditional method, self-assessment is a critical procedure during the process of autonomous learning which requires the students themselves to be more involved and play a more significant role in assessing their learning and outcome by implementing appropriate methodologies and using the learning goals that they have set before against which to decide whether their learning has achieved certain goals or not. The most significant point of self-assessment lies in not the constant reminding the students of taking control of their own learning but providing the students with the platform of meeting with the different, diverse and characterized learning needs of different individuals.

The most reliable and practical ways that can be taken are setting up one's own portfolios and self-examination. Setting up one's portfolios means one should consciously collect all the possible records such as documents, videos, pictures and information and etc. that have been used or taken during a period of time. These longitudinally collected documents can provide a clear and vivid picture of how much progress one has made and by reviewing these records one can clearly be aware of where he/she is and how much effort he/she should take to accomplish the set goals. This can be more effective when it is applied to improve one's communication skills, while self-examination is more suitable in acquiring the knowledge of cross-cultural communication. The mastery of language knowledge, foreign customs and taboos and so on can be better and easily measured by examination.

\section{5) Modify existing learning strategies according to the self-assessment}

The ultimate goal of assessment is to find out whether the existing learning strategies work well. In order to better serve the learning goals, the students should seriously take the result, feedback and information gathered from the self-assessment, and must be ready to make any modification to the existing strategies if needed. After the modification, one cycle of the learning process is formed and it repeats again.

\section{REFERENCES}

[1] Benson. P. \& P. Voller. (1997). Autonomy and Independence in Language Learning. London: Longman.

[2] Cheng Xiaotang (1999). On Autonomous Learning. Research on Cultivation of Course Ability. 9, 32-39.

[3] Claire E. Weinstein (1987). Fostering Learning Autonomy Through the Use of Learning Strategies. Journal of Reading, Vol. 30, No. 7, Making Readers Independent (Apr., 1987), pp. 590-595. International Reading Association.

[4] Dickinson, L. (1987). Self-instruction in language Learning, pp.9-12. Cambridge: Cambridge University Press.

[5] Eccles, J. (1983). Expectancies, values and academic behaviors. In J. T. Spence, Achievement and achievement motives (pp. 75-146). San Francisco: Freeman.

[6] Holec, H. (1981). Autonomy and Foreign Language Learning, pp. 2-5. Oxford: Pergamon Press.

[7] http://en.wikipedia.org/wiki/Cross-cultural_communication (accessed 20/11/3013).

[8] http://www.ewbs.com/descr.html(accessed 20/11/3013).

[9] McShane, Steven L \& Mary Ann Von Glinow (2003).Organizational Behavior. New York: McGraw-Hill.

[10] Pintrich, P. R. (1999). The role of motivation in promoting and sustaining self-regulated learning.pp. 459-470. International Journal of Educational Research. Oxford: Pergamon Press.

[11] Spitzberg, B. H. (1994).A Model of Intercultural Communication Competence [A]. In L. A. Samovar \& R. E. Porter (eds.) Intercultural Communication: A Reader (7th edition) [C]. Wadworth, Inc., 1994: 347 - 359.

[12] Spitzberg, B.H. and Cupach, W.R. (1984). Interpersonal Communication Competence. Beverly Hills, CA: Sage Publications.

[13] The Ministry of Education of China. (2007). College English Curriculum Requirement. Beijing: Tsinghua University Press.

[14] Wenden, A. (1998). Learner Strategies for Learner Autonomy, pp.41-41. London: Prentice Hall.

[15] Wright, T. (1987). Roles of Teachers and Learners, pp.12.Oxford: Oxford University Press.

Fangpeng Gai was born in Laiyang, China in 1979. He is currently lecturer in the School of Foreign Language Studies, Shandong Jiaotong University, Jinan, China. His research interests include English teaching and cross culture studies and this article is among his research outcomes when he was a visiting scholar as the University of Memphis, Memphis, USA from July $20^{\text {th }}, 2013$ to January $7^{\text {th }}, 2014$. 